\title{
Theory of dark-state polariton collapses and revivals
}

\author{
S. D. Jenkins, D. N. Matsukevich, T. Chanelière, A. Kuzmich, and T. A. B. Kennedy \\ School of Physics, Georgia Institute of Technology, Atlanta, Georgia 30332-0430, USA
}

(Received 27 September 2005; published 22 February 2006)

\begin{abstract}
We investigate the dynamics of dark-state polaritons in an atomic ensemble with ground-state degeneracy. A signal light pulse may be stored and retrieved from the atomic sample by adiabatic variation of the amplitude of a control field. During the storage process, a magnetic field causes rotation of the atomic hyperfine coherences, leading to collapses and revivals of the dark-state polariton number. These collapses and revivals should be observable in measurements of the retrieved signal field, as a function of storage time and magnetic field orientation.
\end{abstract}

\section{DOI: $10.1103 /$ PhysRevA.73.021803}

A quantum memory element consisting of an ensemble of atoms, with efficient coupling to a signal light field, represents a node in several quantum network architectures [1-4]. A dark-state polariton (DSP) is a collective excitation, with light field and atomic spin wave parts, in which the relative size of the light and matter contributions can be varied by changing the amplitude of a control laser field [1]. In connection with atomic memories, DSPs offer the possibility for efficient transfer of information between a light carrier and an atomic medium, with programmable storage of the excitation in the atomic spin coherence. The storage and subsequent retrieval of the signal field component of the DSP can be achieved by the extinction and subsequent reactivation of the control field after a given storage time. Experimental demonstrations of "stopped light" can be understood in terms of the concept of DSP in just this way [5-7].

In a recent work the storage and retrieval of single photons using an atomic ensemble-based quantum memory was reported, with a storage time conjectured to be limited by inhomogeneous broadening in the ambient magnetic field [8]. During the storage, the DSP consists entirely of atomic spin wave, and in order to understand its dynamics in a magnetic field it is necessary to properly account for the atomic level degeneracy and the signal and control field polarizations. In particular for alkali atoms, which have nonzero nuclear spin, the electronic levels have hyperfine structure. In this case we must define a more general form of DSP field operator than that of a simple lambda configuration, in which the atomic spin wave part corresponds to a particular superposition of hyperfine coherences of the ground electronic level. These coherences are, in turn, intimately related to the phenomenon of electromagnetically induced transparency (EIT) [9-12].

We shall see that in a magnetic field the temporal evolution of the DSP reveals a series of collapses and revivals due to the evolution of its spin wave component during the storage phase of the process. We predict that the collapses and revivals should be directly observable in measurements of the retrieved signal field as a function of storage time.

We develop the theory of EIT in a degenerate atomic medium with ground levels $g$ and $g^{\prime}$, and excited level $e$, which have energies $\hbar \omega_{g} \equiv 0, \hbar \omega_{g^{\prime}}$, and $\hbar \omega_{e}$, respectively (Fig. 1). The Zeeman states of level $g$ are written $|g, m\rangle$, where $-F_{g} \leqslant m \leqslant F_{g}$; similar definitions hold for the other levels. All $N$ atoms are assumed to be initially prepared in level $g$
PACS number(s): 42.50.Dv, 03.65.Ud, 03.67.Mn

without polarization, i.e., the density matrix of atom $\mu$ is $\hat{\rho}_{\mu}=\Sigma_{m} p|g, m\rangle_{\mu}\langle g, m|$ where we write $p=1 /\left(2 F_{g}+1\right)$. The density of atoms in the sample is assumed to be constant. The atoms experience a uniform magnetic field $\mathbf{B}$ oriented at an angle $\theta$ with respect to the light propagation $z$ axis. The magnetic field-atom interaction $\hat{V}_{B}=\mu_{B} \mathbf{B} \cdot \Sigma_{s=g, g^{\prime}, e} g_{s} \hat{\mathbf{F}}_{\mathbf{s}}$, where $\hat{\mathbf{F}}_{s}$ is the projection of the atomic angular momentum operator onto level $s$ and $g_{s}$ is the corresponding Landé $g$ factor. The magnetic field induces a Larmor spin precession that is primarily important in the storage phase, when the signal field amplitude is zero. In a pure three state system, a magnetic field has been used to manipulate the phase of a stored light pulse [7]. We note that in prior work collapses and revivals of single-atom Zeeman coherences have been observed $[13,14]$.

We proceed by generalizing the perturbative treatment of Fleischhauer and Lukin [15] to include the degenerate atomic level scheme and the presence of a magnetic field. We assume the number of photons contained in the signal pulse is much less than the number of atoms in the sample, and we retain only terms up to first order in the signal field amplitude. In this limit, we can neglect the populations of levels $e$ and $g^{\prime}$, as well as the coherences between these levels. Furthermore, for an initially unpolarized sample in level $g$, the ground-state populations and Zeeman coherences, as op-

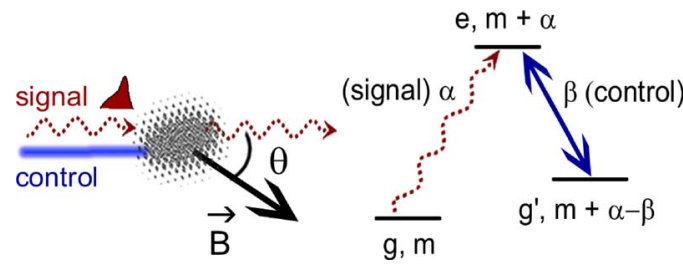

FIG. 1. (Color online) On the left, a diagram shows an atomic ensemble interacting with copropagating signal and control fields. The signal (helicity $\alpha$ ), resonant on the $g \leftrightarrow e$ transition, is stored and subsequently retrieved by variation of a control field (helicity $\beta$ ), which resonantly couples levels $g^{\prime}$ and $e$. A constant magnetic field $\mathbf{B}$, oriented at an angle $\theta$ from the propagation axis, rotates the atomic coherences during the storage. For each state $|g, m\rangle$ in level $g$, there is either an associated $\Lambda$ configuration, as shown on the right, or an unconnected one, as discussed in the text. The signal connects the states $|g, m\rangle$ and $|e, m+\alpha\rangle$, while the control field drives transitions between $|e, m+\alpha\rangle$ and $\left|g^{\prime}, m+\alpha-\beta\right\rangle$. 
posed to hyperfine coherences, are unaffected by the signal, control, and magnetic fields. Our treatment can be extended to an initially spin-polarized atomic sample, as we will report in a separate publication. In this weak signal limit $[1,15]$, the signal field, which we assume propagates in the positive $z$ direction, and atomic coherence operators satisfy the quantum Langevin equations

$$
\begin{gathered}
\left(\frac{\partial}{\partial t}+c \frac{\partial}{\partial z}\right) \hat{\Phi}_{\alpha}=i N \kappa^{*} \sum_{m=-F_{g}}^{F_{g}} C_{m \alpha} \hat{Q}_{e m+\alpha}^{g m}, \\
\frac{\partial}{\partial t} \hat{Q}_{g^{\prime} m^{\prime}}^{g m}=i \Omega(t) C_{m^{\prime} \beta}^{\prime} \hat{Q}_{e^{\prime m} m^{\prime}+\beta}^{g m}, \\
\left(\frac{\partial}{\partial t}+\frac{1}{2} \Gamma_{e}\right) \hat{Q}_{e_{m^{\prime}}^{g m}}^{g m}-\hat{F}_{e^{\prime} m^{\prime}}^{g m} \\
=i \Omega(t) C_{m^{\prime}-\beta, \beta}^{\prime} \hat{Q}_{g^{\prime} m^{\prime}-\beta}^{g m}+i \delta_{m+\alpha, m^{\prime}} \kappa p C_{m \alpha} \hat{\Phi}_{\alpha},
\end{gathered}
$$

where the slowly varying dimensionless electric field of helicity $\alpha= \pm 1$ is given by $\Phi_{\alpha}(z, t)$ $\equiv i \Sigma_{k} \hat{a}_{k, \alpha} \exp \left[i\left(q z+\omega_{e} t\right)\right], \quad q=k-\left(\omega_{e} / c\right), \quad$ and the collective atomic coherence is defined $\hat{Q}_{s^{\prime} m^{\prime}}^{s m}(z, t)$ $\equiv\left(1 / N_{z}\right) \sum_{\mu=1}^{N_{z}} \hat{\sigma}_{s m, s^{\prime} m^{\prime}}^{(\mu)} \exp \left[-i\left(\omega_{s}-\omega_{s^{\prime}}\right)(t-z / c)\right] \quad[16]$, where $\mu=1, \ldots, N_{z}, \hat{\sigma}_{s m, s^{\prime} m^{\prime}}^{(\mu)}$ is the $\mu$ th atom hyperfine coherence operator, and $N_{z}=N d z / L$ is the number of atoms between $z$ and $z+d z ; L$ is the length of the sample. The control field is assumed to have the circular polarization $\beta= \pm 1$ and real Rabi frequency $\Omega(t)$, which is a specified function of time, and $\kappa$ is the coupling constant for the probe transition. We adopt the shorthand for the Clebsch-Gordan coefficients $C_{m \alpha} \equiv C_{m \alpha m+\alpha}^{F_{g} 1 F_{e}}$ and $C_{m \beta}^{\prime} \equiv C_{m \beta m+\beta}^{F_{g^{\prime}} 1 F_{e}}$; it is useful to define $R_{m \alpha}(\beta)=C_{m \alpha} / C_{m+\alpha-\beta, \beta}^{\prime}$.

The decay rate of level $e$ is denoted by $\Gamma_{e}$ and $\hat{F}_{e^{\prime} m^{\prime}}^{g m}$ is a corresponding quantum noise operator. The coupling of the atoms to the uniform magnetic field can be taken into account by the addition of appropriate commutators with the interaction $\hat{V}_{B}$ in the atomic equations.

We first establish some standard features of EIT with our model. The propagation of a classical (coherent) signal through the medium is found by dropping the quantum noise operator, and replacing the field and coherence operators with their respective expectation values. For a constant amplitude control field, the linear susceptibility for the signal field of angular frequency $\omega$ is found to be

$$
\chi_{\alpha}(\Delta) \approx \frac{c d}{2 \omega_{e} L} \sum_{m} \frac{\Gamma_{e} \Delta X_{m \alpha}^{2}\left(\Omega^{2} C_{m+\alpha-\beta, \beta}^{\prime 2}-\Delta^{2}+i \Delta \Gamma_{e} / 2\right)}{\left(\Omega^{2} C_{m+\alpha-\beta, \beta}^{\prime 2}-\Delta^{2}\right)^{2}+\left(\Delta \Gamma_{e} / 2\right)^{2}},
$$

where $\Delta \equiv \omega-\omega_{e}$ is the detuning of the signal from atomic resonance, and $X_{m \alpha} \equiv C_{m \alpha} / \sqrt{\left(2 F_{e}+1\right) / 3}$. The dimensionless quantity $d$ is the optical thickness, which is defined such that $\exp (-d)$ is the on-resonance intensity transmittance in the absence of a control field, and can be expressed as

$$
d=2 \pi w \frac{N}{A}\left(\frac{c}{\omega_{e}}\right)^{2} \frac{2 F_{e}+1}{2 F_{g}+1},
$$

where $w$ is the fraction of atoms in excited level $e$ that spontaneously decay into ground level $g$, and $A$ is the crosssectional area of the ensemble. When a control field is present, an EIT window exists provided that the ClebschGordan coefficients $C_{m+\alpha-\beta, \beta}^{\prime}$ do not vanish for any $-F_{g} \leqslant m \leqslant F_{g}$ for which $C_{m \alpha} \neq 0$. If, however, $C_{m+\alpha-\beta, \beta}^{\prime}=0$, and $C_{m \alpha} \neq 0$, it means that there is an excited state $|e, m+\alpha\rangle$ not coupled by the control field to a state in the ground level $g^{\prime}$, i.e., there is an unconnected lambda configuration. The subset of atoms initially in the state $|g, m\rangle$ would absorb the signal field and spontaneously emit radiation as if there were no control field present. In order for EIT to exist, one must make a judicious choice of atomic levels and signal and control field polarizations.

Assuming a choice of polarizations that supports EIT, we are able to generalize the adiabatic treatment of Ref. [1] to Eq. (1) to derive the DSP operator for helicity $\alpha$, with control field polarization $\beta$

$$
\hat{\Psi}_{\alpha}(z, t)=\frac{\Omega(t) \hat{\Phi}_{\alpha}(z, t)-N \kappa^{*} \sum_{m} R_{m \alpha}(\beta) \hat{Q}_{g^{\prime} m+\alpha-\beta}^{g m}(z, t)}{\sqrt{\Omega(t)^{2}+N p|\kappa|^{2} \sum_{m}\left|R_{m \alpha}(\beta)\right|^{2}}} .
$$

As in Ref. [1], this operator obeys the simple propagation equation $\left(\partial / \partial t+v_{g} \partial / \partial z\right) \hat{\Psi}_{\alpha}(z, t)=0$ with the reduced group velocity $v_{g}=c \Omega^{2} /\left(\Omega^{2}+N p|\kappa|^{2} \Sigma_{m}\left|R_{m \alpha}(\beta)\right|^{2}\right)$ that can be adiabatically controlled by the time-dependent variation of $\Omega(t)$. From the definition of $\hat{\Psi}$, we see that as $\Omega$ goes to zero, the wave excitation stops propagating and transforms into a particular linear combination of hyperfine coherences $\sim \sum_{m} R_{m \alpha}(\beta) \hat{Q}_{g^{\prime} m+\alpha-\beta}^{g m}(z, t)$. This nontrivial result arises from the treatment of the full degeneracy of the atomic ensemble; only this combination of hyperfine coherences is adiabatically transformed into the signal field via the control field retrieval process. Orthogonal combinations of hyperfine coherences couple to optical coherences in the presence of the control field and result in excited state spontaneous emission; we will refer to these as the bright-state polariton (BSP) component [15]. It is also possible that some population of atoms remains trapped in the ground states, and is unaffected by the control field.

In order to demonstrate the importance of the dark state polariton in the signal storage and retrieval process in a magnetic field, we numerically solve Eqs. (1) for a coherent signal field. We thus calculate the expectation values of the spin wave coherences $\left\langle\hat{Q}_{g^{\prime} m+\alpha-\beta}^{g m}(z, t)\right\rangle$ and the signal field, allowing us to determine the DSP and BSP components. This is accomplished by defining a vector space of $2 F_{g}+2$ dimensions, with orthonormal basis vectors $\mathbf{e}_{m}$, each corresponding to a hyperfine coherence $\hat{Q}_{g^{\prime} m+\alpha-\beta}^{g m}$, and $\mathbf{e}_{\Phi}$ corresponding to the signal field. We define the coherence vector $\mathbf{v} \equiv\left\langle\hat{\Phi}_{\alpha}\right\rangle \mathbf{e}_{\Phi}+\Sigma_{m} \sqrt{N / p}\left\langle\hat{Q}_{g^{\prime} m+\alpha-\beta}^{g m}(z, t)\right\rangle \mathbf{e}_{m}$. We note that this is 

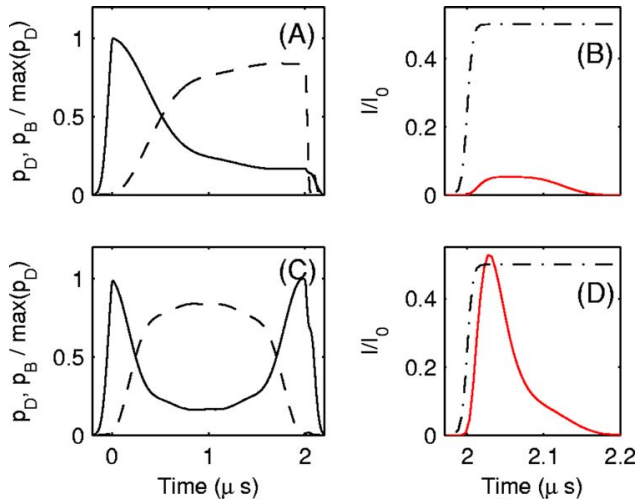

FIG. 2. (Color online) Numerical results illustrate the storage and retrieval of a signal pulse from an atomic ensemble as described in the text. We show results for two values of the magnetic field oriented along the $z$ axis. Panels (A) and (B) correspond to a magnetic field $B=0.267 \mathrm{G}$, so that the signal is stored for $T_{L} / 4$, where $T_{L}=8 \mu \mathrm{s}$ is the Larmor period. Panels (C) and (D) show results for $B=0.535 \mathrm{G}$, corresponding to a signal storage time of $T_{L} / 2$, where $T_{L}=4 \mu \mathrm{s}$. The signal field intensity transmittance $I(t) / I_{0}$ (solid line) and control field Rabi frequency (dot-dashed line), displayed in arbitrary units, are shown in panels (B) and (D). Panels (A) and (C) display scaled dark-state $p_{D}$ (solid line) and bright-state $p_{B}$ (dashed line) polariton components, as explained in the text. In panel (B) the ratio of retrieved to input signal pulse energy is $4.38 \%$ while in (D) the ratio is $25.09 \%$.

not normalized since its magnitude is dependent on both the time-dependent signal and control fields. Associated with the DSP we define a vector $\mathbf{u}_{\Psi} \equiv \Omega \mathbf{e}_{\Phi}+\sqrt{N p} \kappa \Sigma_{m} R_{m \alpha}(\beta) \mathbf{e}_{m}$ and the corresponding unit vector $\mathbf{e}_{\Psi}=\mathbf{u}_{\Psi} /\left\|\mathbf{u}_{\Psi}\right\|$. We then determine the DSP component $p_{D}=\left|\mathbf{e}_{\Psi} \cdot \mathbf{v}\right|^{2}$, and BSP component $p_{B}=\left\|\mathbf{v}-\mathbf{e}_{\Psi} \mathbf{e}_{\Psi} \cdot \mathbf{v}\right\|^{2}$.

As an example of signal storage and retrieval we consider an atomic sample of ${ }^{85} \mathrm{Rb}$, in which the control field and signal field polarizations are chosen equal $\alpha=\beta=1$, and the optical thickness $d=8$, Fig. 2. The atomic levels $g, g^{\prime}$, and $e$ correspond to the $5 S_{1 / 2} F=2,3$, and $5 P_{3 / 2} F=3$ levels of the $D_{2}$ line, respectively. The spontaneous decay rate $\Gamma_{e} /(2 \pi)=5.98 \mathrm{MHz}$. The incident signal field has a Gaussian envelope of full width half maximum $120 \mathrm{~ns}$, and the peak enters the 3-mm-long sample at $t=-60 \mathrm{~ns}$. The control field has a constant Rabi frequency $\Omega=1.5 \Gamma_{e}$ until it is smoothly turned off at $t=0$ over a period of $20 \mathrm{~ns}$, when a fraction of the signal field is converted into hyperfine coherences of the atomic spin wave. The excitation is stored from $0 \leqslant t \leqslant 2 \mu \mathrm{s}$ in the presence of the magnetic field, before the control field is reactivated, and the signal field retrieved. In Fig. 2, panels (A) and (B), the magnetic field is chosen so that the storage time corresponds to a quarter of a Larmor period $T_{L} \equiv 2 \pi \hbar /\left|g_{g} \mu_{B} \mathbf{B}\right|$, while in panels (C) and (D), the storage time is $T_{L} / 2$. In panel (A) $p_{D}$ grows as the signal pulse arrives at the point of observation, and reaches a peak when the control field is switched off. It then decays during the storage phase, due to Larmor precession of the hyperfine coherences in the applied magnetic field, which causes the corresponding growth of $p_{B}$. When the control field is reactivated, $p_{B}$ decays rapidly due to excited level coupling and subsequent spontaneous emission, though $p_{D}$ remains finite as the spin wave coherence of the DSP is converted into the forward propagating signal field; the retrieved signal field intensity is illustrated in panel (B). In panel (C), where the storage time is $T_{L} / 2, p_{D}$ undergoes a complete revival. The energy of the retrieved signal field shown in panel (D) is therefore much larger, by a factor of 5.73, than that in panel (B). This is in good agreement with the DSP theory for retrieval efficiency discussed later, which predicts that the retrieved signal energy of panel (D) should be 5.53 times that in panel (B). These results demonstrate the importance of the adiabatic concept of DSP for a realistic experimental scenario. The retrieved signal field directly reflects the DSP dynamics in the magnetic field.

We can predict the retrieval efficiency of a stored signal pulse by tracking the population of the DSP as it evolves under the influence of the magnetic field. To compute the polariton population, it is convenient to consider the Fourier components of the DSP. We express the polariton annihilation operator for the mode of wave number $q$ as

$\hat{\Psi}_{\alpha}(q, t)=\frac{i \Omega(t) \hat{a}_{k, \alpha}-\sqrt{N p} \kappa^{*} \sum_{m} R_{m \alpha}(\beta) \hat{S}_{g^{\prime} m+\alpha-\beta}^{g m}(q, t)}{\sqrt{\Omega^{2}+N p|\kappa|^{2} \sum_{m} R_{m \alpha}(\beta)^{2}}}$,

where

$$
\begin{aligned}
\hat{S}_{g^{\prime} m^{\prime}}^{g m} \equiv & (1 / \sqrt{N p}) \sum_{\mu} \hat{\sigma}_{g m, g^{\prime} m^{\prime}}^{(\mu)} \\
& \times \exp \left\{-i\left[q z_{\mu}+\left(\omega_{g}-\omega_{g^{\prime}}\right)\left(t-z_{\mu} / c\right)\right]\right\}
\end{aligned}
$$

is a collective spin wave annihilation operator of wave number $q$. These operators obey quasibosonic commutation relations $\left[\hat{S}_{g^{\prime} m_{1}^{\prime}}^{g m_{1}}(q), \hat{S}_{g^{\prime} m_{2}^{\prime}}^{g m_{2}}\left(q^{\prime}\right)\right]=\delta_{m_{1} m_{2}} \delta_{m_{1}^{\prime} m_{2}^{\prime}} \delta_{q q^{\prime}}+O(1 / N)$, and the DSP operators therefore also obey $\left[\Psi_{a}(q), \Psi_{a^{\prime}}^{\dagger}\left(q^{\prime}\right)\right]$ $=\delta_{q q^{\prime}} \delta_{a a^{\prime}}+O(1 / N)$. During the storage, the evolution of the spin wave operators is given by $\hat{S}_{m+\alpha-\beta}^{g m}(q, t)$ $=\sum_{m_{1}=-F_{g}}^{F_{g}} \sum_{m_{2}=-F_{g^{\prime}}}^{F_{g^{\prime}}} \mathcal{D}_{m_{1} m}^{(g) \dagger}(t) \mathcal{D}_{m+\alpha-\beta, m_{2}}^{\left(g^{\prime}\right)}(t) \hat{S}_{g^{\prime} m_{2}}^{g m_{1}}(q, 0), \quad$ where $\mathcal{D}_{m, m^{\prime}}^{(s)}(t) \equiv\left\langle s, m\left|\exp \left(-i g_{s} \boldsymbol{\Omega}_{\mathbf{B}} \cdot \hat{\mathbf{F}} t\right)\right| s, m^{\prime}\right\rangle$ is the matrix element of the rotation operator for states in hyperfine level $s$, and $\boldsymbol{\Omega}_{B} \equiv \mu_{B} \mathbf{B} / \hbar$. Using the bosonic commutation relations for the spin wave operators, we can calculate the number of polaritons $\left\langle N_{\alpha}\left(t_{s}\right)\right\rangle=\left\langle\Sigma_{q} \hat{\Psi}_{\alpha}^{\dagger}\left(q, t_{s}\right) \hat{\Psi}_{\alpha}\left(q, t_{s}\right)\right\rangle$ as a function of storage time $t_{s}$ for an arbitrary DSP quantum state created in the storage process. In the limit of infinite control field amplitude, this converts into the total number of photons in the retrieved signal field $\sum_{k} \hat{a}_{k, \alpha}^{\dagger} \hat{a}_{k, \alpha}$. We therefore derive an expression for the signal retrieval efficiency as the fraction $f_{\alpha}\left(t_{s}\right) \equiv\left\langle\hat{N}_{\alpha}\left(t_{s}\right)\right\rangle /\left\langle\hat{N}_{\alpha}(0)\right\rangle$

$$
\begin{aligned}
f_{\alpha}\left(t_{s}\right)= & \mid \sum_{m_{1} m_{2}} \frac{R_{m_{1} \alpha}(\beta) R_{m_{2} \alpha}(\beta)}{\sum_{m}\left|R_{m \alpha}(\beta)\right|^{2}} \mathcal{D}_{m_{2} m_{1}}^{(g)}\left(t_{s}\right) \\
& \times\left.\mathcal{D}_{m_{1}+\alpha-\beta, m_{2}+\alpha-\beta}^{\left(g^{\prime}\right) \dagger}\left(t_{s}\right)\right|^{2} .
\end{aligned}
$$

In Fig. 3, we display the $f_{\alpha}\left(t_{s}\right)$ as a function of $t_{s}$ for a variety 


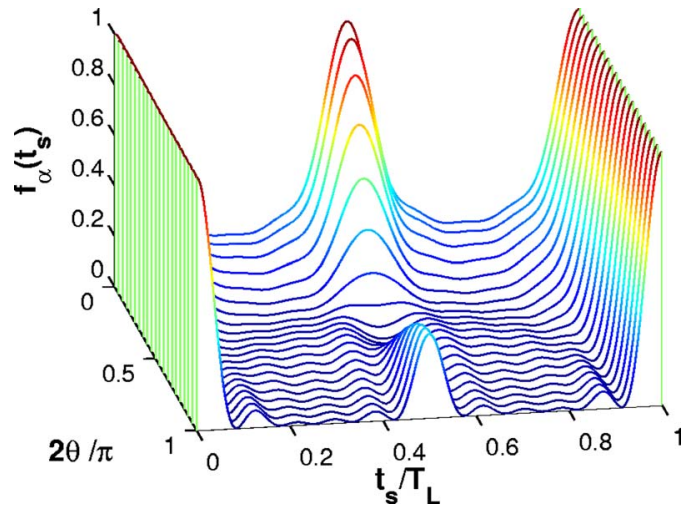

FIG. 3. (Color online) The DSP population fraction $f_{\alpha}\left(t_{s}\right)$ calculated for orientations of the magnetic field $0 \leqslant \theta \leqslant \pi / 2$ over one Larmor period. These results illustrate collapses and revivals whose features are dependent on $\theta$. The atomic configuration and field polarizations $\alpha$ and $\beta$ are described in the text.

of magnetic field orientations. We again consider an ensemble of ${ }^{85} \mathrm{Rb}$ atoms with the same choice of atomic configuration and field polarizations discussed earlier. For $t_{s} \ll T_{L}$, we observe a collapse in the polariton population, yielding an approximate retrieval efficiency of $f_{\alpha}\left(t_{s}\right)$ $\approx \exp \left[-\eta_{\alpha}^{2}\left(\Omega_{L} t_{s}\right)^{2} / 2\right]$, where the collapse rate $\eta_{\alpha}$ depends on the angle, $\theta$, between the magnetic field and the propagation axis. For $\theta=0$, we find

$$
\eta_{\alpha}^{2}(\theta=0)=4 \sum_{m_{1}, m_{2}} \frac{\left|R_{m_{1} \alpha}(\beta) R_{m_{2} \alpha}(\beta)\right|^{2}}{\left(\sum_{m}\left|R_{m \alpha}(\beta)\right|^{2}\right)^{2}}\left(m_{1}-m_{2}\right)^{2} .
$$

With the approximation $g_{g}=-g_{g^{\prime}}$, valid for ground level alkalis, it is clear that the system undergoes a revival to the initial state after a complete Larmor period, and thus the signal retrieval efficiency should equal the zero storage time value. Depending on the orientation of the magnetic field, we observe also a partial revival at half the Larmor period. For a magnetic field oriented along the $z$ axis, the system dynamics are relatively simple. Each hyperfine coherence $\hat{S}_{g^{\prime} m+\alpha-\beta}^{g}$ merely picks up a phase factor that oscillates at $m+(\alpha-\beta) / 2$ times twice the Larmor frequency, thus returning the system to its initial state at half the Larmor period. In this case, the partial revival is actually a full revival. On the other hand, for $\theta=\pi / 2$, a rotation through half the Larmor period causes the coherence transformation $\hat{S}_{g^{\prime} m+\alpha-\beta}^{g m} \rightarrow \hat{S}_{g^{\prime}-(m+\alpha-\beta)}^{g-m}$ up to an overall phase factor. As a result, for the choice of equal field polarizations $(\alpha=\beta)$, the retrieval efficiency at half the Larmor period simplifies to $\left[\Sigma_{m} R_{m \alpha}(\alpha) R_{-m, \alpha}(\alpha) / \Sigma_{m}\left|R_{m \alpha}(\alpha)\right|^{2}\right]^{2}$, resulting in a partial revival. For other orientations of the magnetic field, particularly for $\theta=\pi / 4$, the revival at half the Larmor period is suppressed. This reflects the more complicated dynamics of the individual spin coherences $\hat{S}_{g^{\prime} m^{\prime}}^{g m}$, each of which transforms into a superposition of all $\left(2 F_{g}+1\right)\left(2 F_{g^{\prime}}+1\right)$ spin coherences, with complex time-dependent coefficients governed by the rotation matrices. Stated physically, there is a strong destructive interference between the various spin coherences when $\theta \approx \pi / 4$.

We have developed a theory of the DSP as a mechanism to store and retrieve light pulses in a degenerate unpolarized atomic medium. The role of the DSP and its connection to storage retrieval efficiency have been verified by full numerical solutions of the propagation equations for a classical incident signal field. In the presence of a magnetic field, we have demonstrated that the DSP population undergoes collapses and revivals during the pulse storage time. We predict that this polariton dynamics is directly reflected in the signal pulse retrieval efficiency. Our results may find applications in quantum communication and computation approaches that utilize quantum memories [2,17-19].

Note added. Recently, the collapses and revivals were observed [20], in excellent agreement with the predictions of this paper.

This work was supported by NASA, Office of Naval Research, National Science Foundation, Research Corporation, Alfred P. Sloan Foundation, and Cullen-Peck Chair.
[1] M. Fleischhauer and M. D. Lukin, Phys. Rev. Lett. 84, 5094 (2000).

[2] L.-M. Duan, M. D. Lukin, I. J. Cirac, and P. Zoller, Nature (London) 414, 413 (2001).

[3] M. D. Lukin, Rev. Mod. Phys. 75, 457 (2003).

[4] M. Saffman and T. G. Walker, Phys. Rev. A 72, 049903(E) (2005).

[5] D. F. Phillips et al., Phys. Rev. Lett. 86, 783 (2001).

[6] C. Liu, Z. Dutton, C. H. Behroozi, and L. V. Hau, Nature (London) 409, 490 (2001).

[7] A. Mair et al., Phys. Rev. A 65, 031802(R) (2002).

[8] T. Chanelière et al., Nature (London) 438, 833 (2005).

[9] S. E. Harris, Phys. Today 50, 736 (1997).

[10] M. O. Scully and M. S. Zubairy, Quantum Optics (Cambridge University Press, Cambridge, 1997).
[11] H. Y. Ling, Y.-Q. Li, and M. Xiao, Phys. Rev. A 53, 1014 (1996).

[12] K.-J. Boller, A. Imamoğlu, and S. E. Harris, Phys. Rev. Lett. 66, 2593 (1991).

[13] J. Schmiedmayer et al., J. Phys. II 4, 2029 (1994).

[14] G. A. Smith et al., Phys. Rev. Lett. 93, 163602 (2004).

[15] M. Fleischhauer and M. D. Lukin, Phys. Rev. A 65, 022314 (2002).

[16] F. Haake et al., Phys. Rev. A 20, 2047 (1979).

[17] H.-J. Briegel, W. Dür, J. I. Cirac, and P. Zoller, Phys. Rev. Lett. 81, 5932 (1998).

[18] E. Knill, R. Laflamme, and G. Milburn, Nature (London) 409, 46 (2001).

[19] Y. L. Lim et al., Phys. Rev. A 73, 012304 (2006).

[20] D. N. Matsukevich et al., Phys. Rev. Lett. 96, 033601 (2006). 